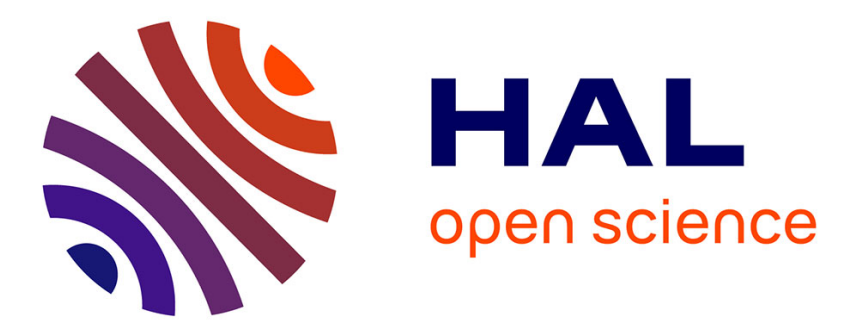

\title{
Adaptive physical resource block design for enhancing voice capacity over LTE network in PMR context
}

Manh Cuong Nguyen, Hang Nguyen, Eric Georgeaux, Philippe Mège, Laurent Martinod

\section{- To cite this version:}

Manh Cuong Nguyen, Hang Nguyen, Eric Georgeaux, Philippe Mège, Laurent Martinod. Adaptive physical resource block design for enhancing voice capacity over LTE network in PMR context. PIMRC 2016: 27th International Symposium on Personal, Indoor and Mobile Radio Communications, Sep 2016, Valencia, Spain. pp.1 - 5, 10.1109/PIMRC.2016.7794632 . hal-01446292

\section{HAL Id: hal-01446292 \\ https://hal.science/hal-01446292}

Submitted on 25 Jan 2017

HAL is a multi-disciplinary open access archive for the deposit and dissemination of scientific research documents, whether they are published or not. The documents may come from teaching and research institutions in France or abroad, or from public or private research centers.
L'archive ouverte pluridisciplinaire HAL, est destinée au dépôt et à la diffusion de documents scientifiques de niveau recherche, publiés ou non, émanant des établissements d'enseignement et de recherche français ou étrangers, des laboratoires publics ou privés. 


\title{
Adaptive Physical Resource Block Design for Enhancing Voice Capacity over LTE in PMR Context
}

\author{
Manh-Cuong Nguyen and Hang Nguyen ${ }^{1} \quad$ Eric Georgeaux, Philippe Mege and Laurent Martinod ${ }^{2}$
}

\begin{abstract}
In this paper, we introduce a new Adaptive Physical Resource Block Design for Enhancing Voice Capacity over LongTerm Evolution (LTE) Downlink in Professional Mobile Radio (PMR) Context. In this method, we reorganize the structure of the Physical Resource Block (PRB) to optimize the voice capacity of LTE downlink in the PMR Context. Available PRBs in each subframe is reorganized into a number Sub Physical Resource Blocks. The number of control symbols can be selected flexibility. The proposed method allows reducing both data overhead and control overhead issues for Voice over LTE downlink in PMR context. On average, the voice capacity gain were shown to have about $117.2 \%$ in comparison with the standard LTE.

Index Terms-Adaptive PRB, control symbol, voice capacity, control overhead, data overhead, LTE, PMR
\end{abstract}

\section{INTRODUCTION}

The current Professional Mobile Radio (PMR) systems which are used for public safety, such as TETRA, APCO25 suffer from slow data transfer [11], [9]. Long Term Evolution (LTE) technology takes its place as the choice for the broadband PMR to satisfy the user demands of public safety in the future. In the public safety systems, the performance in terms of voice capacity is one of the strict requirements. However, LTE is not yet optimal for low bit rate voice communication in PMR context because of the data overhead and control overhead issues [5]. Therefore, the voice capacity is not increased as expected of the PMR service providers. In order to deploy LTE as the solution for the next generation of PMR network, some adaptations have to be considered to reduce both data and control overhead.

Factors thought to be influencing voice capacity of VoLTE have been explored in several studies. The current solutions on data overhead of voice over LTE pays particular attention to reduce the size of header protocols (e.g. [4], [12]). However, in the PMR context the difference between the LTE packet size and the PMR voice payload is the main factor that causes the data overhead. Our research shows that the average size of the LTE packet is about three times greater than the PMR voice payload. In addition, state-of-the-art methods expose many

\footnotetext{
${ }^{1}$ Institut Mines-Telecom, Telecom SudParis Samovar Laboratory, UMR 5157, CNRS, Evry, France \{manh_cuong.nguyen, hang.nguyen $\} @$ it-sudparis.eu

${ }^{2}$ Department of Security and Communication Solutions CASSIDIAN (an EADS Company), Elancourt, France \{eric.georgeaux, philippe.mege, laurent.martinod\}@cassidian.com
}

This work was accomplished in the framework of the collaborative project SOAPS.2, labeled by the Systematic PARIS-REGION Cluster difficulties to obtain the improvement of the Voice over LTE (VoLTE) capacity in PMR context. The solutions for reducing data overhead do not take into account the signal overhead whenever evaluating the system performance and on the other hand, the existing researches on control overhead such as Semi-persistent scheduling (SPS) [8], TTI-bundling [6], packet bundling [10] do not take into account the data overhead. Therefore, we propose a new Adaptive Physical Resource Block Design for Enhancing Voice Capacity over Long-Term Evolution (LTE) Donwlink in Private Mobile Radio (PMR) Context. In this method, we reorganize the structure of the Physical Resource Block (PRB) to optimize the voice capacity of LTE downlink in the PMR Context. The available PRBs in each subframe is reorganized into a number Sub Physical Resource Blocks (subPRBs). The number of control symbols can be selected flexibility. The proposed method allows reducing both data overhead and control overhead issues for VoLTE downlink in PMR context. The details of this method is presented in section II. The performance of our method is evaluated in section III. Section IV will give the conclusion and some perspectives.

\section{Proposed Adaptive Physical Resource Design}

\section{A. Proposed Sub Physical Resource structure}

In LTE [3], [7], [2], [1] one pair of Physical Resource Blocks (PRBs) is the smallest User Assignment Unit. However, the smallest LTE packet size is still too large in case that low bit rate voice communication is transmitted in high Modulation and Coding Scheme (MCS). This is the main factor that affects the data overhead of VoLTE in PMR context. Therefore, the main idea of the proposed method is to reorganize the structure of the PRB of LTE to optimize the voice capacity of VoLTE in case of PMR context but not affect the operations of the system.

In order to ensure the integrity of processing for control signals such as Reference Signal (RS), Primary Synchronization Channel (PSS), Secondary Synchronization Channel (SSS)...These control signals are organized in the same way with LTE standards. It means that one radio frame still includes 10 subframes of $1 \mathrm{~ms}$. Each subframe consists of two slots. Each slot in turn consists seven (normal cyclic prefix) or six symbols (extended cyclic prefix). The number and location of control channels such as PSS, SSS, RS.. are unchanged. The difference is that in the new design, the available REs for data in each subframe is reorganized into a number Sub Physical 


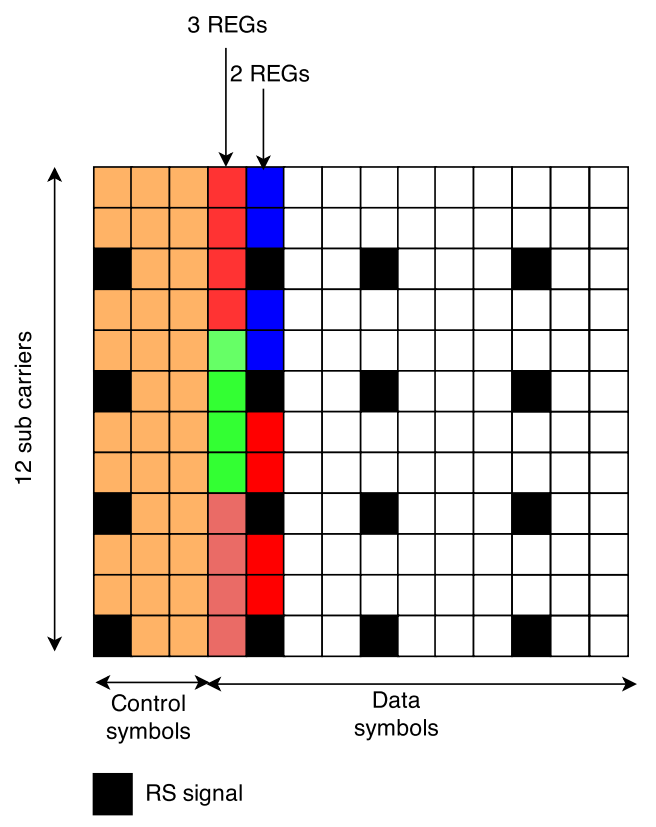

Fig. 1. REG structure

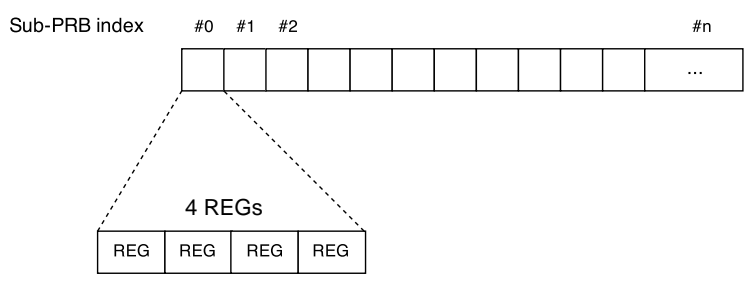

Fig. 2. Adaptive Phycical Resource Block

Resource Blocks (SubPRBs). Each SubPRB consists of four Resource Element Group (REG). Each Resource Element Group (REG) contains four consecutive Resource Elements (RE) or four REs separated by a cell-specific Reference Signal (RS). The structure of SubPRB is similar to the structure of Control Channel Element (CCE) for the control channel with adaptations. SubPRB structure is illustrated in Figure 1.

In addition, in the new design, instead of limiting the number of symbols for control channels from 1 to 3 symbols, the new design allows a flexible choice of the number of symbols so that the system can obtain the maximum voice capacity. Figure 3 illustrates the model of our proposed system. There is a slight change in the last step. The symbols after passing the Layer mapping/precoding are mapped into subPRBs instead of PRBs. Therefore, the major issues to be considered is how to determine the size of SubPRB and the number of symbols used for control channel to maximize the voice capacity. In LTE, the base scheduler is Fully Dynamic (FD) scheduler. In the FD scheduler, each data packet needs to associate with a Layer 1 (L1) control signaling (a Physical Downlink Control Channel). LTE uses Physical Downlink
Control Channel (PDCCH) to carry all allocation information for both downlink and uplink shared channels. These symbols for control channels are organized in Resource Element Group and Control Channel Element (CCE). One REG consists of four consecutive REs or four REs separated by one Reference Signal. One CCE comprises nine REGs. To build the PDCCH, LTE uses a number of consecutive CCEs called CCE aggregation level. The CCE aggregation level can be one, two, four or eight. The aggregation level depends on the DCI size and the effective coding rate. There are four PDCCH formats (PDCCH format $0, \mathrm{PDCCH}$ format $1, \mathrm{PDCCH}$ format 2, $\mathrm{PDCCH}$ format 3) that correspond to four aggregation levels. PDCCH carries Downlink Control Information (DCI). We know that the number of control channels depends on the size of the DCI and the code rate. The size of DCI in turn depends on the number of available PRBs (in case of LTE) or available SubPRB (in our case). Therefore, the choice of size SubPRB influences the number of available control channels. If the size of SubPRB is too small, the number of bits need to be used for resource allocation in the corresponding DCI is increased. However, if the size of SubPRB is too big, the gap between LTE packet size and the PMR voice payload will be increased. In case of PMR with voice target codec 2450 bps, the optimal value of subPRB size is about four REGs. Therefore, in the proposed SubPRB design, we propose that the one SubPRB can consist of four REGs (see Figure 2). The number of symbols for control channel are chosen to optimize the voice capacity of VoLTE in PMR context.

\section{B. System Model}

We consider the number of available control channel $\left(N_{C}\right)$ is a function of the number of allowed resource blocks of a defined bandwidth $N_{B W}$, the number of symbols used for PDCCH channels $n_{c}$, number of active UEs in the cell with the aggregation level $A_{i j}$ that is need to be used to transmit PDCCH for $U E_{i}$ at $j^{t h}$ TTI, voice payload of UE $S_{p}$,

$$
N_{C}=f\left(N_{B W}, n_{c}, S_{p},\left[A_{i j}\right]\right)
$$

We consider the number of available data channels $\left(N_{D}\right)$ is a function of $N_{B W}, n_{c}, S_{p}$ number of active UEs in the cell with Modulation and Coding Scheme assigned $M C S_{i j}$

$$
N_{D}=f\left(N_{B W}, n_{c}, S_{p}, C P,\left[M C S_{i j}\right]\right)
$$

The voice capacity $N_{V}$ at the $j^{\text {th }}$ TTI is defined as:

$$
N_{V}=\min \left(N_{C}, N_{D}\right)
$$

At the $j^{\text {th }}$ TTI, choose $n_{c}$ so that:

$$
\underset{n_{c}, n_{k}}{\operatorname{argmax}} f\left(n_{c}\right)=\left\{n_{c} \mid \forall x: f(x) \leq f\left(n_{c}\right)\right\}
$$

The voice capacity in a period $\left\{t_{1}, t_{2}\right\}$ is defined as:

$$
\text { Voicecapacity }=\sum_{t_{1}}^{t_{2}} N_{V}
$$




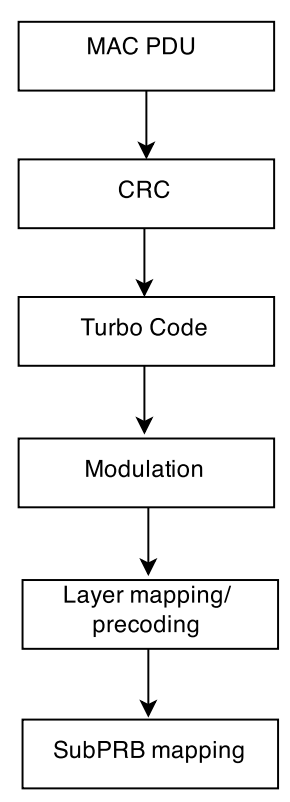

Fig. 3. SubPRB mapping
TABLE II

SIMULATION PARAMETERS

\begin{tabular}{|l|l|}
\hline Parameter & Value \\
\hline Bandwidth & $1.4 \mathrm{MHz}, 3 \mathrm{MHz}, 5 \mathrm{MHz}$ \\
\hline LTE & Type 1 FDD \\
\hline Codec & AMBE 2450 bps \\
\hline $\mathrm{Ng}$ & 1 \\
\hline CRC & 24 bits \\
\hline Cyclic Prefix & Normal \\
\hline Number of antenna & 2 \\
\hline
\end{tabular}

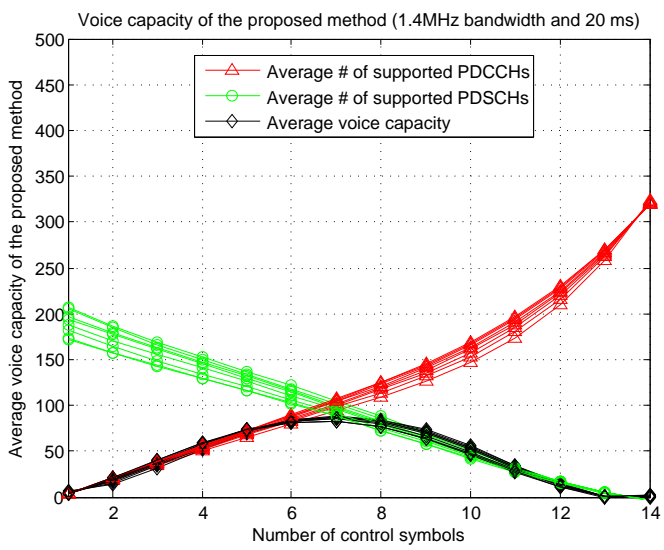

Fig. 4. Control capacity of the proposed method (1.4 MHz bandwidth and 20ms)

1.4 MHz bandwidth in $20 \mathrm{~ms}$ simulation. As Table III shows, there is a significant voice capacity gain (54 for standard LTE and 91 for the proposed method) between the two methods. Comparing the two results, it can be seen that the proposed method allows a more flexible and optimal LTE packet size than the existent standard LTE. The standard LTE is not optimal the packet size in case of PMR context. This leads to a decrease number of available data packets and control packets. Meanwhile, the proposed method allows a choice packet size and the number of symbols for control channel more flexible so that the proposed method can chose an optimal value for improving voice capacity.

Table IV and Table V present the voice capacity gain for the case of $3 \mathrm{MHz}, 5 \mathrm{MHz}$ bandwidths. For the $3 \mathrm{MHz}$ bandwidth, the voice capacity gain is about $134.7 \%$ and for the $5 \mathrm{MHz}$ bandwidth the voice capacity gain is about $133.3 \%$.

In our simulation, we also apply several SubPRB sizes (from one to eight REGs) get the optimal value of SubPRB size for the PMR voice codec of 2450 bps. Figures 4, 5 and 6 show the relation between the number of symbols for control channel with the number of average supported data packets average supported control packets and the voice capacity for the three corresponding $1.4 \mathrm{MHz}, 3 \mathrm{MHz}, 5 \mathrm{MHz}$ bandwidths for different SubPRB sizes. The results show that the optimal value number of symbols for control channel is about 5 to 7 symbols and the SubPRB contains about four REGs.

Table III compares the average voice capacity obtained from Physical Resource Block design (standard LTE) and from Adaptive Physical Resource Block design (our method) for 
TABLE III

VOICE CAPACITY GAIN OF THE PROPOSED METHOD FOR 1.4 MHZ IN 20 MS

\begin{tabular}{|c|c|c|c|c|c|c|c|c|c|c|c|c|c|c|}
\hline Number of control symbols & 1 & 2 & 3 & 4 & 5 & 6 & 7 & 8 & 9 & 10 & 11 & 12 & 13 & 14 \\
\hline Average supported control packets of FD & 7 & 31 & 54 & $\mathrm{X}$ & $\mathrm{X}$ & $\mathrm{X}$ & $\mathrm{X}$ & $\mathrm{X}$ & $\mathrm{X}$ & $\mathrm{X}$ & $\mathrm{X}$ & $\mathrm{X}$ & $\mathrm{X}$ & $\mathrm{X}$ \\
\hline Average supported data packets of FD & 84 & 84 & 84 & $\mathrm{X}$ & $\mathrm{X}$ & $\mathrm{X}$ & $\mathrm{X}$ & $\mathrm{X}$ & $\mathrm{X}$ & $\mathrm{X}$ & $\mathrm{X}$ & $\mathrm{X}$ & $\mathrm{X}$ & $\mathrm{X}$ \\
\hline Average supported control packets of new method & 6 & 26 & 46 & 67 & 87 & 107 & 127 & 148 & 168 & 188 & 208 & 229 & 249 & 269 \\
\hline Average supported data packets of new method & 169 & 157 & 140 & 129 & 115 & 99 & 86 & 72 & 57 & 44 & 28 & 14 & 7 & 0 \\
\hline Average voice capacity of FD & \multicolumn{14}{|c|}{54} \\
\hline Average voice capacity of new method & \multicolumn{14}{|c|}{99} \\
\hline Gain & \multicolumn{14}{|c|}{$83.5 \%$} \\
\hline
\end{tabular}

X: Standard LTE not support

TABLE IV

VOICE CAPACITY GAIN OF THE PROPOSED METHOD FOR 3 MHZ IN 20 MS

\begin{tabular}{|c|c|c|c|c|c|c|c|c|c|c|c|c|c|c|}
\hline Number of control symbols & 1 & 2 & 3 & 4 & 5 & 6 & 7 & 8 & 9 & 10 & 11 & 12 & 13 & 14 \\
\hline Average supported control packets of FD & 16 & 54 & 92 & $\mathrm{X}$ & $\mathrm{X}$ & $\mathrm{X}$ & $\mathrm{X}$ & $\mathrm{X}$ & $\mathrm{X}$ & $\mathrm{X}$ & $\mathrm{X}$ & $\mathrm{X}$ & $\mathrm{X}$ & $\mathrm{X}$ \\
\hline Average supported data packets of FD & 208 & 208 & 208 & $\mathrm{X}$ & $\mathrm{X}$ & $\bar{X}$ & $\mathrm{X}$ & $\mathrm{X}$ & $\mathrm{X}$ & $\mathrm{X}$ & $\mathrm{X}$ & $\mathrm{X}$ & $\mathrm{X}$ & $\mathrm{X}$ \\
\hline Average supported control packets of new method & 13 & 46 & 79 & 112 & 146 & 181 & 216 & 253 & 290 & 331 & 375 & 431 & 631 & 681 \\
\hline Average supported data packets of new method & 481 & 446 & 397 & 360 & 325 & 279 & 241 & 201 & 159 & 120 & 80 & 41 & 0 & 0 \\
\hline Average voice capacity of FD & \multicolumn{14}{|c|}{92} \\
\hline Average voice capacity of new method & \multirow{2}{*}{\multicolumn{14}{|c|}{$\frac{216}{134.7 \%}$}} \\
\hline Gain & & & & & & & & & & & & & & \\
\hline
\end{tabular}

X: Standard LTE not support

TABLE V

VOICE CAPACITY GAIN OF THE PROPOSED METHOD FOR 5 MHZ IN 20 MS

\begin{tabular}{|c|c|c|c|c|c|c|c|c|c|c|c|c|c|c|}
\hline Number of control symbols & 1 & 2 & 3 & 4 & 5 & 6 & 7 & 8 & 9 & 10 & 11 & 12 & 13 & 14 \\
\hline Average supported data packets of FD & 347 & 347 & 347 & $\mathrm{X}$ & $\mathrm{X}$ & $\mathrm{X}$ & $\mathrm{X}$ & $\mathrm{X}$ & $\mathrm{X}$ & $\mathrm{X}$ & $\mathrm{X}$ & $\mathrm{X}$ & $\mathrm{X}$ & $\mathrm{X}$ \\
\hline Average supported control packets of new method & 23 & 75 & 129 & 182 & 237 & 293 & 350 & 410 & 470 & 536 & 608 & 695 & 1052 & 1137 \\
\hline Average supported data packets of new method & 805 & 737 & 672 & 597 & 535 & 470 & 403 & 337 & 269 & 202 & 134 & 69 & 0 & 0 \\
\hline Average voice capacity of new method & \multirow{2}{*}{\multicolumn{14}{|c|}{$\frac{350}{33.3 \%}$}} \\
\hline Gain & & & & & & & & & & & & & & \\
\hline
\end{tabular}

$\mathrm{X}$ : Standard LTE not support

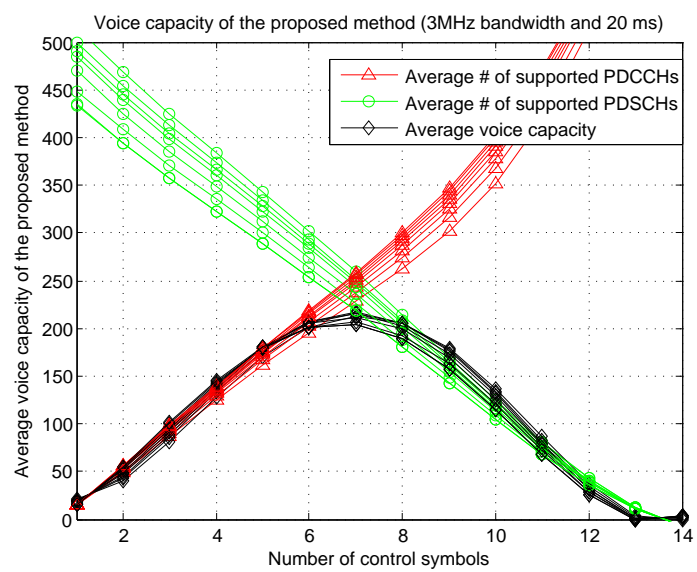

Fig. 5. Control capacity of the proposed method (3 MHz bandwidth and 20ms)

\section{CONCLUSION}

In this paper, we proposed a new Adaptive Physical Resource Design for improving control capacity of LTE Downlink in the PMR context. In this design, the available PRBs in each subframe is reorganized into a number of subPRBs.

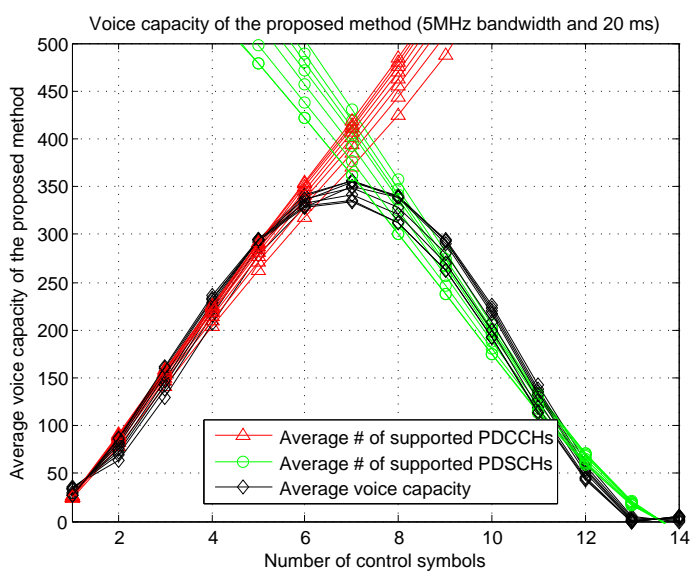

Fig. 6. Control capacity of the proposed method (5 MHz bandwidth and 20ms)

In addition, the number of symbols for control channel are chosen flexibility to maximize the voice capacity. On average, the voice capacity gain were shown to have about $117.2 \%$ in comparison with the standard LTE. Finally, a number of important limitations need to be considered. First, the 
complexity assessment and the energy consumption issue of the new design has not been studied. Second, an assessment of the of Bit Error Rate (BER) should also be considered when the number of supported voice calls is increased.

\section{REFERENCES}

[1] Evolved Universal Terrestrial Radio Access (E-UTRA); Physical layer procedures, 3GPP TS. 36.213 V9.0.1, Dec. 2009.

[2] Evolved Universal Terrestrial Radio Access (E-UTRA); User Equipment (UE) radio transmission and reception, 3GPP TS. 36.101 V9.2.0, Dec. 2009.

[3] D Astély, E Dahlman, A Furuskar, Y Jading, M Lindstrom, and S Parkvall. LTE: the evolution of mobile broadband. Communications Magazine, IEEE, 2009.

[4] C Bormann, C Burmeister, M Degermark, H Fukushima, H Hannu, LE Jonsson, R Hakenberg, T Koren, K Le, Z Liu, et al. RObust Header Compression (ROHC): Framework and four profiles: RTP, UDP, ESP, and uncompressed. Technical report, RFC 3095, July, 2001.

[5] AA Florea, L Martinod, P Mege, and H Nguyen. Multi-layer Realistic Voice Capacity Evaluation in LTE Rel. 9 and Performance Comparison with PMR and GSM. In World Telecommunications Congress (WTC), 2012.
[6] J Han and H Wang. Principle and performance of TTI bundling for VoIP in LTE FDD mode. In Wireless Communications and Networking Conference (WCNC), 2009.

[7] Telesystem Innovations Inc. LTE in a Nutshell: The Physical Layer. 2010.

[8] D Jiang, H Wang, E Malkamaki, and E Tuomaala. Principle and performance of semi-persistent scheduling for VoIP in LTE system. In Wireless Communications, Networking and Mobile Computing (WiCom), 2007.

[9] Xavier Pons Masbernat, Christophe Gruet, Frédéric Fraysse, Serge Contal, and Lirida Naviner. Green solutions for future lte pmr networks. In Computer Aided Modeling and Design of Communication Links and Networks (CAMAD), 2012 IEEE 17th International Workshop on, pages 165-167. IEEE, 2012.

[10] J Puttonen, T Henttonen, N Kolehmainen, K Aschan, M Moisio, and $\mathrm{P}$ Kela. Voice-over-IP performance in UTRA Long Term Evolution downlink. In Vehicular Technology Conference (VTC), 2008.

[11] M Simic. Feasibility of long term evolution (lte) as technology for public safety. In Telecommunications Forum (TELFOR), pages 158-161, 2012.

[12] ER Vale, MA Grivet, and JC Brandao. Reducing the VoIP packet overhead in UMTS air interface. In Adaptive Science \& Technology (ICAST), 2009. 